during sleep (Wada, 1922). Another possibility is that Horlicks may provide amino-acids which are converted into neural transmitters and in this way influence central nervous function. Or the response may be in some way associated with the fat in milk. In this connexion, Fara, Rubenstein, and Sonnenschein (1969) found that milk or $1 \mathrm{ml}$ or corn oil introduced into the duodenum of cats increased the proportion of rapid eye movement sleep. They believe that the phenomenon may be related to the release of cholecystokinin.

That the general state of nutrition may influence sleep patterns is apparent from the work of Crisp and Stonehill (1971), who found that patients successfully treated for anorexia nervosa slept longer with fewer movements than they did before treatment. Whether this change was the result of the state of nutrition or the effect of the size of a recent meal is not apparent.

Experiments are in progress to check our results.

\section{References}

Crisp, A. H., and Stonehill, E. (1971). Fournal of Psychosomatic Research,

Fara, J. W., Rubenstein, E. H., and Sonnenschein, R. R. (1969). Science, 166,110

Kleitman, N., Ramsaroop, A., and Engelmann, T. (1958). Federation Proceedings, 7, 66.

Parish, P. A. (1971). Fournal of the Royal College of General Practitioners, 21, Suppl. No. 4

Wada, T. (1922). Archives de Psychologie, 8, 1.

\title{
Sleep after a Bedtime Beverage
}

\author{
VLASTA BŘEZINOVÁ, IAN OSWALD
}

British Medical fournal, 1972, 2, 431-433

\section{Summary}

Sleep after an inert capsule was compared with sleep after a hot, bedtime, milk-cereal drink (Horlicks). Allnight electrophysiological recordings were made with 18 persons on 10 nights each.

Restlessness during sleep at the end of the night was diminished after Horlicks in a group of 10 young adults. In an older group of eight adults, mean age 55 years, sleep after Horlicks was of longer total duration and was less broken by periods of wakefulness. In the latter group the improvement associated with Horlicks was most apparent late in the night and it increased with repeated administration.

\section{Introduction}

Hypnotic drugs are much in fashion but popular belief has it that there are simpler aids to good sleep. Many people take a drink before bed, mixing proprietary products with hot milk. One such product has for several generations been claimed to promote good sleep-namely, Horlicks, a milk-cereal powder.

Subjective evaluation of sleep quality is unreliable, and we report here an objective study of sleep after Horlicks. The work of Southwell et al. (1972) was known to us, and this permitted the prediction that in young adults sleep after Horlicks would be accompanied by fewer signs of movement at the end of the night. We also used the more general hypotheses that sleep after Horlicks would be of greater duration and less broken. Young adults generally sleep so well that there is little scope for improvement, and so we also studied older people. Broken sleep is an accompaniment of normal ageing (Feinberg, 1968).

Nearly forty years ago Laird and Drexel (1934) reported that the nocturnal sleep of young adults was accompanied by fewer movements after a light meal of cornflakes and milk whereas a heavy meal made sleep more restless. Like Southwell et al. (1972) they measured only the frequency of body movements. Unfortunately this does not allow discrimination between movements made while asleep and move-

Department of Psychiatry of the University of Edinburgh, Royal Edinburgh Hospital, Edinburgh

VLASTA BR̆EZINOVÁ, M.D., PH.D., Research Fellow

IAN OSWALD, M.D., Reader in Psychiatry ments made during episodes of wakefulness. Preconceived ideas about the effect of a food drink might especially reduce motility during wakefulness. We used electroencephalography, which makes possible discrimination between wakefulness and sleep.

\section{Subjects and Methods}

The drink was prepared by mixing about $32 \mathrm{~g}$ of Horlicks with $250 \mathrm{ml}$ of hot milk. As a control we used an inert yellow capsule, intimating that it contained a folk remedy of doubtful efficacy. We considered and rejected the use of water as a control because it would be more rapidly absorbed, leading to a brisk diuresis, rapid bladder filling, and positive disturbance of sleep. We also rejected plain milk because if no difference were found between it and the Horlicks drink we should not know whether both had been without action on sleep or whether each had had a positive but similar effect.

The drink or capsule was administered just before lights-out at about 22.30 hours and always by the same investigator (V.B.), whose own attitude towards Horlicks was one of slightly amused scepticism. Subjects rose at 07.30 hours.

The first group consisted of 10 healthy volunteers (six men, four women) aged 20-30 years (mean 22 years). The women were in the early part of their menstrual cycle.

The older group consisted of eight healthy volunteers (three men, five women) aged 42-66 years (mean 55 years). Four had a professional background, including one distinguished physiologist aged 66. Three subjects were housewives of non-professional background and the eighth was the husband of one.

Subjects were asked not to eat after 19.00 hours and to avoid any irregularities in their normal daily routines. Alcohol was prohibited.

Each subject slept in comfortable circumstances in the sleep laboratory on 10 nights spread over 12-36 days (mean 21 days) according to their availability. In the case of the older people the average interval between laboratory nights was 1.7 days.

On five laboratory nights Horlicks was taken and on five the placebo. The order of administration was based on the sequence HP PHHPPHHP and its obverse. The subjects were studied in concurrent pairs, one always differing from the other in the experimental condition.

All the nights were recorded electrophysiologically, but in accordance with custom in our research unit and a written plan before the study the first two nights were treated as adaptation nights and their results were not included in the main analysis of data. 


\section{MEASURES}

Whole-night sleep was recorded electrophysiologically by means of bipolar leads to give two channels of eye movement from oblique derivations, one channel of electroencephalogram (E.E.G.) from frontal and parieto-occipital electrodes in the $m$ dline, and one channel of submental muscle tension (E.M.G.). Paper speed was $15 \mathrm{~mm} / \mathrm{sec}$.

The records were scored visually in 20 -second epochs, the scorer being unaware of the experimental condition, whether placebo or Horlicks. The E.E.G. sleep stages of Rechtschaffen and Kales (1968) were utilized, sleep onset being taken as the first stage 2 sleep. The raw scores were processed by computer to give an analysis of the night in terms of wakefulness, orthodox sleep stages $1,2,3$, and 4, paradoxical sleep, the transitions between each stage, and the distribution of events during the night in terms of each hour of accumulated sleep. Thus it might take 65 minutes to complete the first hour of sleep if there had been two intervening episodes of wakefulness lasting two and three minutes each. The first six hours of sleep might be completed six hours 30 minutes after sleep onset owing to several intervening awakenings of a total duration of $\mathbf{3 0}$ minutes. In this sense we refer below to hours of sleep, whether individual or summed.

In this study additional electrophysiological events were scored-namely, transitory changes in E.M.G. activity consistent with body movement. This was done to permit some comparison with the movement data of Southwell et al. (1972). Preliminary trials of waking subjects, who made limb or body movements while submental E.M.G. was being recorded, prompted us to score all transitory increases of E.M.G. when these rose to a level three times as high as the previous level and lasted more than two seconds, whether or not accompanied by movement artefacts. There were two categories-(a) without major slow movement artefacts, and $(b)$ with major slowmovement artefacts indicative of change of body axis. Not more than one event was scored per 20-second epoch.

On their first and last nights subjects answered short questionnaires about their sleeping and feeding habits and their attitude to Horlicks.

\section{Results}

The adaptation nights being ignored, means from the four placebo nights and the four Horlicks nights for each individual were computed and the differences between them found. The group results were then subjected to statistical evaluation by using Friedman's two-way analysis of variance (Siegel, 1956) as a non-parametric test.

\section{YOUNG ADULT GROUP}

The prediction that the young adult subjects would show fewer signs of movement during sleep at the end of the night after Horlicks was tested. No difference of trend was noticed between the two categories of movement event so these were combined. All subjects on all nights had attained a minimum of seven hours' sleep. In the seventh hour mean movement events after placebo numbered 14.5 but after Horlicks only $11 \cdot 6$, and nine of the 10 subjects averaged fewer movement events after Horlicks $\left(\chi_{r}^{2}=6.40, P<0.02\right)$. Movements during episodes of wakefulness were, of course, excluded in defining an hour of sleep. Alternatively, when the last three hours of sleep completed by each individual were considered there was a mean of 38.3 events after Horlicks and 42.9 after placebo. Eight of the 10 subjects had fewer movements after Horlicks in their last three hours of sleep, giving $\chi_{\mathrm{r}}^{2}=3.60$ and borderline statistical significance $(P<0.055)$.

No other features of sleep showed significant differences in this group of subjects. Certain features, however, are worthy of mention. Delay to first sleep onset was $27 \cdot 1$ minutes after placebo and 31.5 minutes after Horlicks. After Horlicks the women had increased total sleep, earlier sleep onset, fewer episodes of wakefulness or drowsiness, and a shift of stages 3 and 4 sleep from the early hours towards the middle hours of sleep.

\section{OLDER AGE GROUP}

On average the older people slept less than the young, with a mean duration of only 455.1 minutes compared with 484.6 minutes $(t=5.32$, D.F. $=16, P<0.01)$, and their inter- and intra-individual variance was higher for nearly all measures of sleep.

Seven of the eight subjects had longer mean total sleep after Horlicks. The differences in minutes between the individuals own means on placebo and Horlicks were $+28.8,-30.7$, $+35 \cdot 2,+16 \cdot 6,+1 \cdot 6,+18 \cdot 5,+6 \cdot 9$, and $+10 \cdot 4$. The mean duration of sleep was 439.6 minutes after placebo and 450.5 minutes after Horlicks $\left(x_{\mathrm{r}}^{2}=4.50, P<0.05\right)$.

All subjects on all nights attained a minimum of six hours' sleep. After Horlicks seven of the eight subjects spent less time in intervening wakefulness in the attainment of those six hours. The mean differences from placebo in minutes for the individuals were $-30.8,-20.8,-37.0,-13.4$, nil, $-30.5,-0.3$, and $-9 \cdot 3$. The difference from placebo is illustrated in the chart and was significant $\left(\chi_{\mathrm{r}}^{2}=6 \cdot 13, \mathrm{P}<0.02\right)$.



Sleep of older people after placebo or Horlicks. Sleep was significantly less broken after bedtime Horlicks $(P<0.02)$. Successive completed hours of sleep are shown, each point being based on 32 placebo or 32 Horlicks nights.

The advantage in favour of Horlicks was present throughout the night. In the first three hours of sleep the mean duration of intervening wakefulness was 14.2 minutes on placebo and only 8.4 minutes on Horlicks, but variance was high and the difference was not significant. In the second three hours of sleep mean intervening wakefulness was 15.5 minutes on placebo and only 3.6 minutes on Horlicks $\left(\chi_{r}^{2}=6.13, P<0.02\right)$.

Since the differentiation of a brief period of wakefulness from a brief period of stage 1 sleep (drowsiness) is at times imprecise it is important to consider stage 1 sleep. In the first six hours of sleep there was a mean duration of stage 1 of 32.7 minutes after placebo and $30 \cdot 2$ minutes after Horlicks (not significant). Combining wakefulness and stage 1 sleep for the first six hours the reduction after Horlicks was significant $\left(\chi_{-r}^{2}=4.50\right.$, $P<0.05)$. Seven of the eight subjects spent less time in combined wakefulness and stage 1 sleep after Horlicks.In the second three hours of sleep wakefulness and stage 1 combined were significantly decreased after Horlicks $\left(\chi_{\mathrm{r}}^{2}=4.50, \mathrm{P}<0.05\right)$.

There were no other significant differences in sleep measures between the two conditions. Mean delay to first sleep onset was 19.9 minutes on placebo and 19.1 minutes on Horlicks. Movement events were fewer after Horlicks in the late night but the variance was high. The mean number of spontaneous awake- 
nings in the first six hours was $2 \cdot 1$ on placebo and only 1.2 on Horlicks. Only one of the eight subjects had a greater incidence of awakenings when on Horlicks.

\section{EFFECT OF SERIAL ADMINISTRATION}

In the case of the older group there was a significant trend with time in the association between Horlicks and diminished wakefulness and stage 1 sleep. The adaptation nights were included for this analysis. There had been no significant difference between Horlicks and placebo nights on the two adaptation nights, but when difference scores were computed to express the difference between all five pairs of adjacent nights, where one night of each pair was placebo and the other Horlicks, a strong trend was found towards greater advantage to Horlicks with succeeding experiences, and Friedman's analysis of variance indicated that this was significant $\left(x_{\mathrm{r}}^{2}=11 \cdot 3\right.$, D.F. $=$ 4, P $<0.025$ ).

\section{SUBJECTS' ATTITUDES}

Altogether 10 of the 18 subjects, including six older ones, started with the expressed belief that Horlicks could improve sleep. The remainder were neutral in attitude. After completing the experiment five subjects (including three older ones) believed that Horlicks had improved their sleep and 13 did not. We could find no relation between the expressed individual attitudes and any measured Horlicks-placebo sleep differences or between the latter and the profession of the subjects.

\section{Discussion}

We have offered some confirmation that after Horlicks the sleep of young adults is less restless in the later night. We have found the sleep of older people to be significantly longer and less broken after Horlicks at bedtime. We cannot exclude the possibility that ingrained beliefs were responsible and that the mechanisms were more psychological than physiological, despite reported attitudes. A psychological explanation, however, appears hard to reconcile with the more striking effect of Horlicks in the later night. We would have supposed also that an attitudinal factor would be maximal at entry to the study, whereas our results show a strong serial trend in favour of Horlicks.

The experiment embodied opportunities for learning. Apart from the gradual adjustment to a strange sleeping situation we gave subjects nine hours in bed to allow scope for any length- ening of sleep. They went to bed earlier and stayed therelonger than was their custom. Perhaps Horlicks helped them gradually to take better advantage of the opportunities as the experiment progressed, so facilitating the appearance of a serial trend.

Crisp (1967) ascribed to chronic undernutrition an effect in impairing sleep and reported improvement in sleep after refeeding (Crisp et al., 1971). The relevance of this to the effect of a milk-cereal beverage in adequately nourished persons is not certain. More to the point are the cat experiments of Fara et al. (1969), who showed that milk or corn oil introduced into the cat's duodenum had a sedative effect possibly mediated by cholecystokinin-pancreozymin.

Our results vindicate both popular belief and the statement of Brobeck (1960) that "food is without any doubt the oldest and most widely used tranquillizer." Certainly it is for babies. Perhaps our results should be ascribed to a conditioned response to milk persisting from infancy and strengthened by serial re-evocation during our experiment. If, on the other hand, the observed response is to nutrients further research could indicate whether it is a non-specific response to food, a specific response to milk, or a specific response to the proprietary milkcereal preparation. How ingested nutrients could alter sleep as much as seven hours later provides a particular challenge. Research into the influence of bedtime Horlicks on blood lipids would also be desirable.

The effects of Horlicks are, in our view, weak by comparison with those of hypnotic drugs, but hypnotic drugs become less effective with serial administration, whereas Horlicks became more effective. Perhaps the two would be of comparable efficacy in long-term use.

We are indebted to Beecham Inc., Clifton, New Jersey, U.S.A., for supplies of Horlicks powder, and to Miss Marion Briggs for practical help. The research was financed by Edinburgh University, which received a donation from the Beecham Group Ltd. in aid of research into the relation between sleep and nutrition.

\section{References}

Brobeck, J. R. (1960). In Handbook of Physiology, ed. J. Field, H. W. Magoun, and V. E. Hall, vol. 2, Section 1, Washington, American Physiological Society.

Crisp, A. H. (1967). Fournal of Psychosomatic Research, 11, 117.

Crisp, A. H., Stonehill, E., and Fenton, G. W. (1971). Postgraduate Medical Fournal, 47, 207.

Fara, J. W. Rubinstein, E. H., and Sonnerschein, R. R. (1969). Science, 166,110

Feinbers, I. (1968). Comprehensive Psychiatry, 9, 138.

Feinberg, I. (1968). Comprehensive Psychiatry, 9, 138. ciation, 10, 89.

Rechtschaffen, A., and Kales, A. (1968). Manual of Standardized Terminology, Techniques and Scoring System for Sleep Stages of Human Subjects. Washington, U.S. Public Health Service.

Siegel, S. (1956). Nomparametric Statistics for the Behavioural Sciences. New York, McGraw-Hill.

Southwell, P. R., Evans, C. R., and Hunt, J. N. (1972). British Medical fournal, 2, 429. 